\title{
The initial physiological responses to glucose ingestion in normal subjects are modified by a $\mathbf{3} \mathrm{d}$ high-fat diet
}

\author{
M. B. SIDERY, I. W. GALLEN AND I. A. MACDONALD* \\ Department of Physiology and Pharmacology, University of Nottingham Medical School, \\ Clifton Boulevard, Nottingham NG7 $2 U H$
}

(Received 15 January 1990-Accepted 11 June 1990)

\begin{abstract}
The present study was designed to investigate whether $3 \mathrm{~d}$ of high-fat, low-carbohydrate diet (with normal daily energy intake) affected the metabolic, cardiovascular and thermic responses to an oral glucose load $(1.5 \mathrm{~g} / \mathrm{kg}$ body-weight). Eight normal weight, healthy subjects (five male) consumed diets containing approximately $65 \%$ by energy of carbohydrate (C) or fat (F), each for $3 \mathrm{~d}$. Before and after glucose ingestion, continuous recordings of oxygen consumption and carbon dioxide production were made using indirect calorimetry, and metabolic rate (MR) and respiratory exchange ratio (RER) were calculated. Heart rate, blood pressure and limb blood flow were also measured. There was no significant change in baseline MR following the high-fat diet, but the fasting RER was reduced. The high-fat diet modified the responses to oral glucose, with a greater increase in $\mathrm{MR}(\mathrm{kJ} / \mathrm{min} ; \mathrm{C}+0.38, \mathrm{~F}+0.76)$, and an enhanced plasma insulin response $(\mathrm{mU} / \mathrm{l} ; \mathrm{C}+51 \cdot 5, \mathrm{~F}+\mathbf{8 5} \cdot 0)$. There were no differences between the two diets with respect to the baseline blood glucose levels or the changes after glucose ingestion. Heart rate, systolic and diastolic blood pressures and blood flow responses to the glucose load were similar after the two diets. There was no plasma catecholamine response to glucose ingestion. It can be concluded that a high-fat diet alters the initial thermic response and insulin response but does not alter the other physiological responses to glucose ingestion.
\end{abstract}

Glucose tolerance: Fat intake: Insulin

Ingestion of food is associated with an increase in metabolic rate (MR) (the thermic response) which can be affected by alteration in nutritional state. For example, the thermic response is reduced by a $48 \mathrm{~h}$ fast in healthy subjects (Gallen \& Macdonald, 1990), and by underfeeding in obese subjects (Alban-Davies et al. 1989). The thermic effect of glucose alone has two components, the predictable 'obligatory' energy cost of nutrient storage (Flatt, 1978), and an additional 'facultative' component (Acheson et al. 1984). This facultative component is readily demonstrable after the infusion of glucose and insulin and may be mediated by activation of the sympathetic nervous system, in that it can be abolished by prior administration of the $\beta$-adrenoreceptor antagonist propranolol (Acheson et al. 1983). It has been proposed that reduction in energy intake may cause suppression of facultative thermogenesis and this may considerably affect the thermic response to food ingestion. As fat $(\mathrm{F})$ does not appear to be important in the recruitment or regulation of the thermic effect of food, it is not certain whether in undernutrition it is the reduction in total energy intake or just the reduction in carbohydrate $(C)$ which is responsible for the impaired thermic response to food ingestion seen after fasting or underfeeding. Thus, the present study was designed to see if $3 \mathrm{~d}$ of a high-F, low-C diet with a normal total daily energy intake affected the metabolic, cardiovascular and thermic responses to glucose ingestion. 


\section{METHODS}

Eight healthy (five male), non-obese (mean body mass index 22 , range $20 \cdot 1-25 \mathrm{~kg} / \mathrm{m}^{2}$ ) subjects aged 18-24 years were recruited for the study. None were taking any medication other than the oral contraceptive pill.

All gave written informed consent to the study which was approved by the University of Nottingham Medical School Ethical Committee. All subjects were nutrition students. On the basis of dietary histories, subjects were advised on the composition of foodstuffs eaten. For the high-C period they were given advice as to which foods to eat in large quantities (i.e. rich in $\mathrm{C}$ ) and which to avoid (i.e. high in F). All the subjects recorded the types and amounts of foods eaten and by analysis using computerized food tables, energy derived from $C$ and $F$ was calculated. In the high- $F$ period, subjects avoided foods with high $C$ content and were provided with the appropriate amount of food with a high $F$ content, i.e. double cream, butter, full-fat milk, etc. The percentage of the total energy derived from $\mathrm{C}$ in the high- $\mathrm{C}$ period and $\mathrm{F}$ in the high- $\mathrm{F}$ diet was approximately $65 \%$. Approximately $50 \%$ of the $\mathrm{C}$ consumed in both the diets was starch, the rest being refined. The subjects were studied in the afternoon, $6 \mathrm{~h}$ after a light breakfast whilst on the high- $\mathrm{C}$ diet or the high$\mathrm{F}$ diet. Studies took place in a temperature-controlled room $\left(30^{\circ}\right)$ with the subjects resting supine, wearing a T-shirt and shorts only. On each occasion, female subjects were studied in the first $14 \mathrm{~d}$ of the menstrual cycle to exclude any effects of this on MR (Webb, 1986). Studies in each male subject were performed at least 1 week apart. There was no significant difference between the weights of the subjects after the two dietary periods.

Subjects rested supine for $30 \mathrm{~min}$, during which time intravenous cannulas were inserted under local anaesthetic, and the monitoring equipment attached. Baseline measurements were made for $30 \mathrm{~min}$. The glucose load $(1.5 \mathrm{~g} / \mathrm{kg}$ body-weight $)$ was dissolved in warm $\left(32^{\circ}\right)$ water and consumed within $10 \mathrm{~min}$. Measurements continued for $90 \mathrm{~min}$ after glucose ingestion.

Continuous recordings of oxygen consumption and carbon dioxide production were made using an indirect calorimeter (Fellows \& Macdonald, 1985). From the respiratory gas exchange data, calculations of MR (Weir, 1949) and respiratory exchange ratio (RER) were made. Mean values (10 min) of MR and RER were used for statistical analysis and graphical representation. Heart rate was recorded from an electrocardiogram, and brachial arterial blood pressure was measured by auscultation using a mercury sphygmomanometer taking Korotkoff phase $\mathrm{V}$ as the diastolic blood pressure (DBP). The surface temperature of the skin was measured over the left mid-abdomen, left mid-thigh, left mid-calf, left midshin and dorsum of the left foot using thermocouples lightly taped to the skin, the readings taken from a Comark 6600 microprocessor type thermometer. Right forearm and right calf blood flows were determined by venous occlusion plethysmography using a mercury-inrubber strain gauge (Whitney, 1953).

For blood sampling, a cannula was inserted retrogradely into a vein on the dorsum of the left hand and kept patent with a slow running infusion of saline (154 mmol sodium chloride/1). This hand rested in a warm air box $\left(55-60^{\circ}\right)$ to obtain 'arterialized' venous blood samples (McGuire et al. 1976). Forearm effluent venous blood was obtained from a venous cannula inserted retrogradely into a deep muscle vein of the right forearm (Andres et al. 1954), also kept patent with a saline (154 mmol NaCl/l) infusion. These deep venous samples were taken at the end of each measurement of forearm blood flow, whilst the arterial occlusion cuff at the wrist was still inflated. Immediately after each blood sample, arterialized and deep venous blood glucose concentrations (YSI 23 AM; Yellow Springs Industries, Yellow Springs, USA) were measured. Forearm glucose uptake was calculated from 'arterio-venous' difference in values of blood glucose and the forearm blood flow 

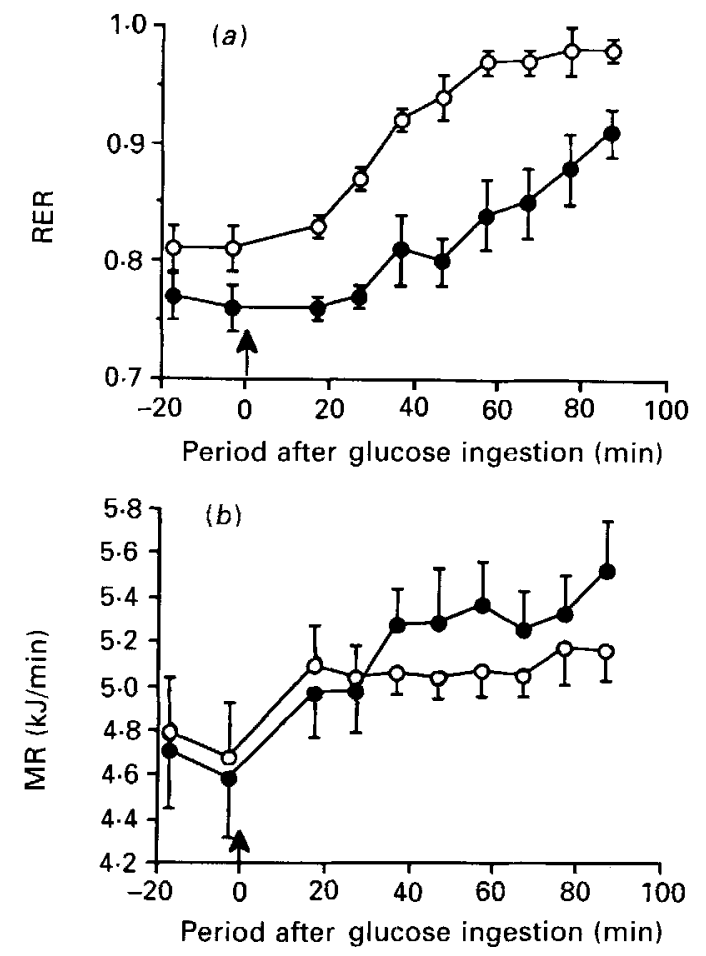

Fig. 1. (a) Respiratory exchange ratio (RER) and (b) metabolic rate (MR) in the high-carbohydrate (O) and highfat (O) fed states before and after glucose ingestion. Period of glucose ingestion indicated by the arrow. Values are means, with their standard errors represented by vertical bars. The high-fat diet reduced RER $(P<0.01$, ANOVA). After glucose ingestion, $M R$ in the high-fat fed state was greater than in the high-carbohydrate fed state $(P<0.01$, ANOVA). The rise in RER was of a similar magnitude in both states $(P<0.05$, ANOVA).

measured at that time. A portion of each arterialized venous blood sample was deproteinized in $0.1 \mathrm{M}$-perchloric acid, the supernatant fraction being stored at $-20^{\circ}$ for later analysis of lactate, glycerol and $\beta$-hydroxybutyrate (BOHB) levels (Lloyd et al. 1978). The remainder of the arterialized blood sample was centrifuged at $4^{\circ}$ and the plasma separated. Plasma $(3 \mathrm{ml})$ was mixed with $100 \mu 1$ EGTA-glutathione (antioxidant) and stored at $-80^{\circ}$ for later determination of noradrenaline concentration using highperformance liquid chromatography with electrochemical detection (Macdonald \& Lake, 1985). Plasma was stored at $-80^{\circ}$ for subsequent determination of insulin concentration.

Statistical analysis of the results was performed by two-way analysis of variance (ANOVA) using the package BMDP. For each variable, analysis was initially undertaken on the baseline value obtained before the ingestion of the glucose to detect any difference between the two diets. For each subject, mean baseline values were then subtracted from the subsequent values to calculate the magnitude of various responses to the glucose load. These responses were further analysed by ANOVA. Values are presented as means. The values reported in the text for responses to the glucose are the maximum changes from mean baseline values. The absolute values are plotted, in Figs. 1-4, as the means with their standard errors of the mean in the baseline period, but after glucose ingestion as the means with their standard errors of the changes in a variable from the baseline. 

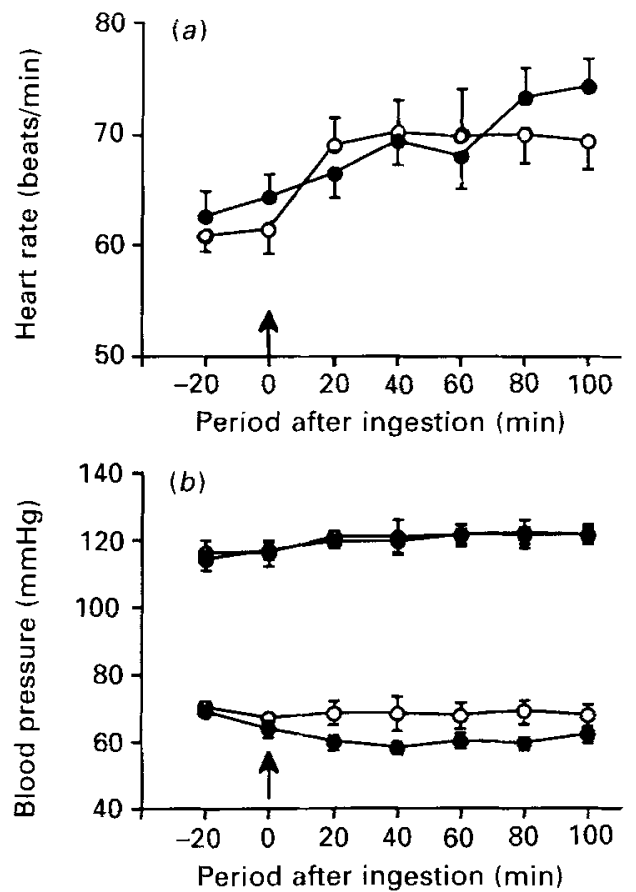

Fig. 2. (a) Heart rate (beats/min), and (b) systolic and diastolic blood pressures (mmHg), in the high-carbohydrate $(O)$ and high-fat $(O)$ fed states before and after food ingestion. Period of glucose ingestion indicated by the arrow. Values are means, with their standard errors represented by vertical bars. After glucose ingestion, the heart rate increase was greater in the high-fat fed state $(P<0.01$, ANOVA). A fall in diastolic blood pressure was seen in the high-fat fed state only $(P<0.01$, ANOVA).

\section{RESULTS}

$M R$ and $R E R$

The baseline MR was similar $(4.75 \mathrm{~kJ} / \mathrm{min}$ after $\mathrm{C}, 4.70 \mathrm{~kJ} / \mathrm{min}$ after $\mathrm{F})$ but RER was significantly reduced by the high-F diet (from 0.81 to 0.76 , treatment effect $\mathrm{f} 1 / \mathrm{f} 21 / 7, F 6 \cdot 3$, $P<0.05$, ANOVA; Fig. 1). After glucose ingestion, there was a significantly different pattern of MR response between the two states, with a greater and more prolonged rise in $\mathrm{MR}$ in the high- $\mathrm{F}$ fed state (mean change over $90 \mathrm{~min}(\mathrm{~kJ} / \mathrm{min}) \mathrm{C}+0 \cdot 38, \mathrm{~F}+0.76$, treatment-time interaction $\mathrm{f} 1 / \mathrm{f} 27 / 49, F 3.63, P<0.005$, ANOVA). The RER rose by similar amounts in the two states $(\mathrm{C}+0 \cdot 17, \mathrm{~F}+0 \cdot 12$, time effect fl/f2 $7 / 49, F 41 \cdot 1$, $P<0.001$, ANOVA).

\section{Haemodynamic responses}

The high-F diet had no significant effect on baseline haemodynamic variables (Figs. 2 and 3). During the baseline period there was a small reduction in DBP on both occasions (C $-3.5 \mathrm{mmHg}, \mathrm{F}-5.3 \mathrm{mmHg}$, time effect $\mathrm{f} 1 / \mathrm{f} 21 / 6, F 8.54, P<0.03$, ANOVA).

Following ingestion of the glucose load there was a greater increase in heart rate in the high- $\mathrm{F}$ fed state $(\mathrm{F}+10.8$ beats $/ \mathrm{min})$ than in the high- $\mathrm{C}$ fed state $(\mathrm{C}+8.9$ beats $/ \mathrm{min}$, treatment-time effect f1/f2 4/28, F 2.59, $P<0.05$, ANOVA). However, after glucose ingestion there was a fall in DBP (but not in systolic blood pressure) in the high-F fed state. One-way ANOVA showed a significant drop in post-prandial DBP (f1/f2 $5 / 30, F 12 \cdot 0$, $P<0.001$, ANOVA). When the DBP responses to glucose in the two states were compared (by two-way ANOVA), there was no difference between them. Forearm blood flow increased after glucose ingestion but the effect was more marked in the high-C fed state 

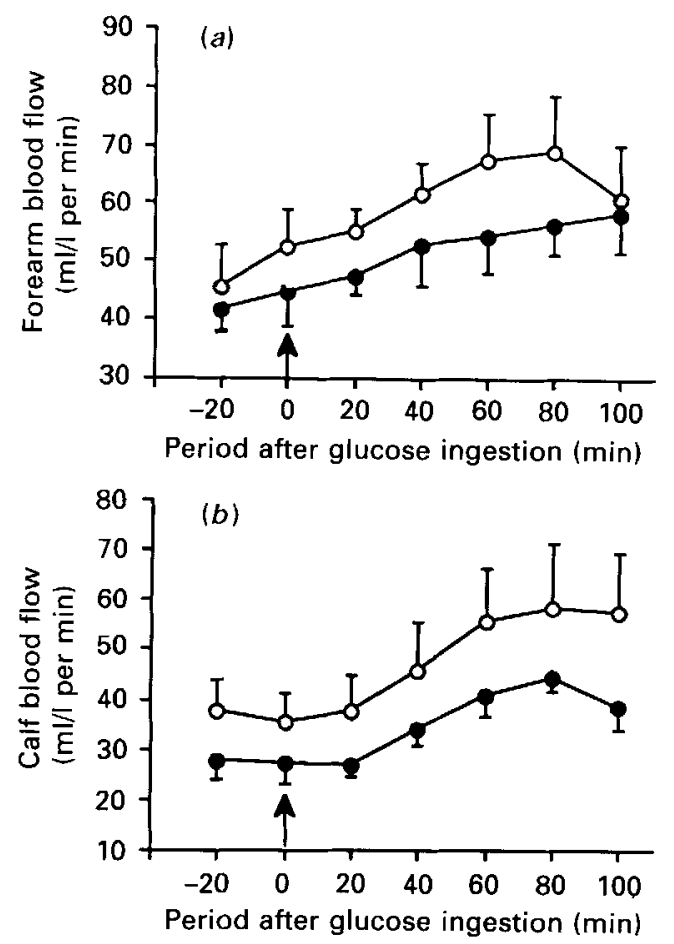

Fig. 3. (a) Forearm blood flow and $(b)$ calf blood flow in the high-carbohydrate $(O)$ and high-fat $(O)$ fed states before and after glucose ingestion. Period of glucose ingestion indicated by the arrow. Values are means, with their standard errors represented by vertical bars. A rise in forearm blood flow was seen in both states, with a greater rise seen in the high-carbohydrate fed state $(P<0 \cdot 01$, ANOVA).

$(\mathrm{C}+22 \mathrm{ml} / 1$ per min, treatment-time effect $\mathrm{f} 1 / \mathrm{f} 24 / 28, F 4 \cdot 1 P<0 \cdot 01$, ANOVA). Calf blood flow increased similarly after glucose ingestion in both states (time effect $\mathrm{f1} / \mathrm{f} 24 / 28$, $F 16.48, P<0.0001$, ANOVA).

\section{Skin temperature}

There was no significant difference in baseline skin temperature at any of the five sites measured after the two diets. After glucose ingestion, there were significant rises in skin temperature of $0.7-2.4^{\circ}$ at four sites, mid-thigh (time effect $\mathrm{f} 1 / \mathrm{f} 23 / 24, F 2.89, P<0.05$, ANOVA), mid-calf (time effect f1/f2 $3 / 18, F 4 \cdot 32, P<0.02$, ANOVA), mid-shin (time effect $\mathrm{fl} / \mathrm{f} 23 / 18, F 3.69, P<0.03$, ANOVA) and dorsum of foot (time effect $\mathrm{f} 1 / \mathrm{f} 23 / 18$, $F 58, P<0.001$, ANOVA), but no significant difference in the response between the two states. No significant increase in skin temperature in either state after glucose was seen at the mid-abdomen measurement site.

Blood glucose and plasma insulin concentrations and forearm glucose uptake

No difference was seen between the two diets in baseline arterialized blood glucose concentration (mmol/1; C 4.2, F 4.2) or plasma insulin (mU/1; C 5.6, F 6.3). After glucose ingestion there were similar rises in arterialized blood glucose $(\mathrm{mmol} / 1 ; \mathrm{C}+5 \cdot 4, \mathrm{~F}+6 \cdot 4$, time effect $\mathrm{fl} / \mathrm{f} 24 / 28, F 13 \cdot 6, P<0.001$, ANOVA). However, there was a greater rise in plasma insulin after glucose ingestion in the high-F fed state (peak increases $(\mathrm{mU} / 1)$, $\mathrm{F}+85 \cdot 0, \mathrm{C}+51 \cdot 5$, treatment-time interaction $\mathrm{f} 1 / \mathrm{f} 24 / 28, F 5 \cdot 2, P<0.005$, ANOVA; Fig. 4). 


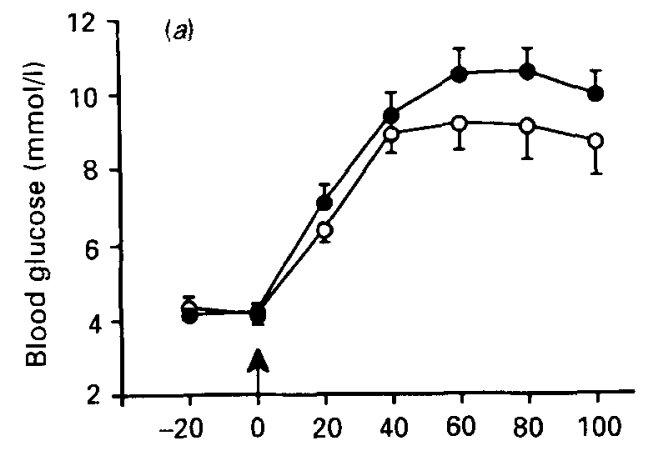

Period after glucose ingestion ( $\mathrm{min}$ )

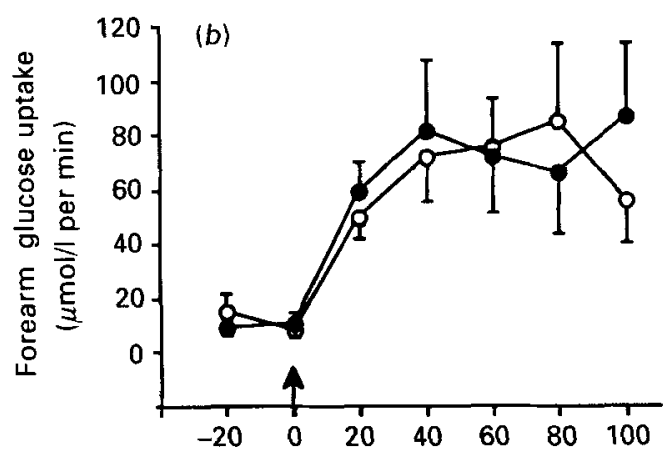

Period after glucose ingestion (min)

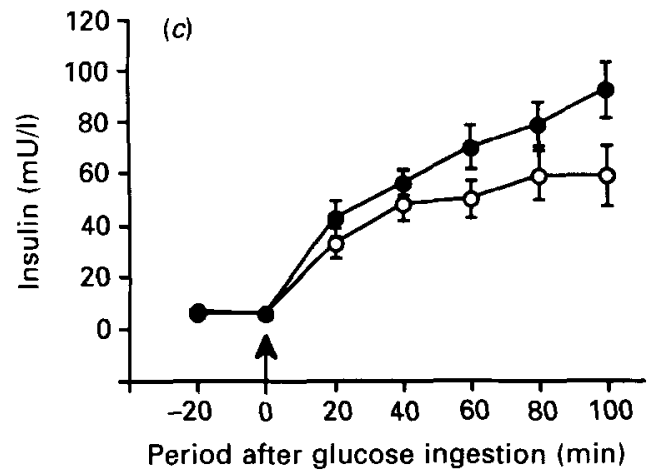

Fig. 4. (a) Blood pressure, (b) forearm glucose uptake and (c) plasma insulin in the high-carbohydrate $(O)$ and high-fat ( $O$ ) fed states before and after glucose ingestion. Period of glucose ingestion indicated by the arrow. Values are means, with their standard errors represented by vertical bars. After glucose ingestion there was a greater rise in insulin concentrations in the high-fat fed state than in the high-carbohydrate fed state $(P<0.021$, ANOVA). Forearm glucose extraction was similar in both states. 
Baseline forearm glucose uptake was similar in both states $(\mu \mathrm{mol} / \mathrm{min}$ per $1 ; \mathrm{C} 11 \cdot 0, \mathrm{~F}$ 10.0). After glucose ingestion, forearm glucose uptake increased by about $75 \mu \mathrm{mol} / \mathrm{min}$ per 1 on both occasions (time effect f1/f2 4/28, F 3.3, $P<0.03$, ANOVA; Fig. 4).

\section{$B O H B$, glycerol and lactate concentrations}

Baseline arterialized venous plasma BOHB concentrations were similar in both states (mmol/1; C 0.26, F 0.23) as were blood glycerol (mmol/1; C 0.08, F 0.06) and lactate $(\mathrm{mmol} / 1 ; \mathrm{C} 0 \cdot 84, \mathrm{~F} 0 \cdot 65)$. After glucose ingestion, similar changes were seen in blood BOHB concentration (mmol/1; C 0.05, F 0.07, time effect, f1/f2 6/30, F 4.5, $P<0.01$ ), lactate concentration (mmol/1; C 1.6, F 1.5, time effect f1/f2 6/36, F 22.9, $P<0.001$, ANOVA) and blood glycerol concentration (C 0.03, F $0.03 \mathrm{mmol} / 1, \mathrm{fl} / \mathrm{f} 26 / 36, F 11.6, P<0.001$, ANOVA).

\section{Plasma noradrenaline}

The high-F diet had no effect on the arterialized venous plasma noradrenaline concentration (nmol/1; C 0.75, F 1.2), and no significant change in plasma noradrenaline concentration was seen after glucose ingestion in either state.

\section{DISCUSSION}

The present study examined the effects of alteration in $C$ intake with maintained energy intake on the thermic, cardiovascular and metabolic response to glucose ingestion.

The high-F diet did not alter resting MR but as expected there was a significant reduction in baseline $R E R$. This reflects an increased use of $F$ as the major metabolic substrate (Lusk, 1924; Elia \& Livesey, 1988). The rise in RER following glucose ingestion was significant but there was no difference in the responses on the two occasions.

After ingestion of glucose, a rise in MR in both states was seen, confirming previous observations of the thermic effect of glucose ingestion (Welle et al. 1980). The design of the present study was such that all subjects were studied first after the high-C diet. Thus, an order effect may have contributed to some of the differences observed. However, the thermic response to glucose after the high-C diet was very similar to the $10 \%$ increase above control values reported by Welle et al. (1980) in subjects given a $100 \mathrm{~g}$ glucose load after a normal mixed diet. Furthermore, the majority of the physiological responses to glucose measured in the present study were similar on the two occasions, the main differences being in the thermic, insulin and heart rate responses.

The greater thermic response to glucose observed after the high-F diet was not due to net lipogenesis from the ingested glucose, as the RER did not rise above 1 in either state and was in fact lower at all times after the high-F diet. The difference in the initial thermic response to glucose may be due to less of a suppression of lipid oxidation following glucose ingestion in the high- $\mathrm{F}$ fed state. With a similar overall thermic response to glucose in the two states this would give rise to an enhanced net thermic response after the high-F diet. Such an effect on lipid oxidation would be consistent with a degree of insulin resistance after the high- $\mathrm{F}$ diet. However, further studies are needed over a longer time-course to determine whether the total thermic response is similarly affected by a high-F diet.

The high-F diet had no significant effect on baseline cardiovascular measurements. After glucose ingestion heart rate rose in both states, but the pattern of response differed. Following the high-C diet heart rate reached a plateau 20 min after glucose ingestion. Following the high-F diet, there was a gradual increase in heart rate after glucose ingestion, which continued throughout the rest of the experiment and resulted in a greater overall response. These different patterns of heart rate change after glucose ingestion are similar 
to the MR responses. Such similarities of response may be coincidental, but it seems probable that the heart rate changes reflect a slightly increased cardiac output necessary to support the small elevation in MR.

DBP fell significantly following glucose ingestion after the high-F diet but not after the high-C diet. It may be that ingestion of glucose arrests the falling baseline DBP after the high- $\mathrm{C}$ diet but not after the high-F diet. Alternatively, the observed reductions in DBP after glucose may be a positive effect of the high-F diet. If this was so, it would imply some additional reduction in peripheral vascular resistance after glucose in this state. By contrast, forearm blood flow and calf blood flow increased significantly after both diets. It is likely that glucose ingestion increases visceral blood flow in both states, with the drop in total peripheral resistance leading to an increase in cardiac output. The highly significant increases in limb blood flow do not, however, lend credence to the hypothesis that only visceral blood flow increases on glucose ingestion. Increases in limb blood flow in response to food ingestion have been recorded in earlier studies (Gallen \& Macdonald, 1990).

Ingestion of a standardized meal increases heart rate in the supine position, with no change in systolic blood pressure, a drop in DBP, an increase in cardiac output in parallel with heart rate, and a drop in total peripheral resistance suggesting a decrease in afterload (Fagan et al. 1986). Increased heart rate observed after food ingestion has been suggested as being mediated by activation of the sympathetic nervous system (Welle et al. 1980). However, in the present study plasma noradrenaline levels failed to change significantly following glucose ingestion. Ingestion of food or glucose gives rise to the secretion of a number of gastrointestinal hormones, as well as insulin. It is established that many of these hormones have cardiovascular effects (Thulin \& Samnegard, 1978) and it is possible that the differences in blood flow and blood pressure seen in the present study are due to alterations in some of these hormones.

Blood glucose concentration and forearm glucose uptake were similar in both dietary states. However, there were higher insulin levels after the high- $\mathrm{F}$ diet, suggesting that the subjects had become a little insulin-resistant. Thus, greater insulin levels were needed to achieve the same forearm glucose uptake rates and blood glucose concentrations. Changes in $\mathrm{C}$ metabolism have been associated with high plasma levels of free fatty acids. Thiebaud et al. (1982) showed that long-chain fatty acid infusion caused a greater inhibition of insulin-mediated glucose storage than of glucose oxidation. In the glucose-fatty acid cycle proposed by Randle et al. (1963), free fatty acids induce a reduction of glucose oxidation. Increased fatty acid oxidation inhibits the enzymes pyruvate dehydrogenase, phosphofructokinase and hexokinase, all of which are involved in glycolysis.

The present study was designed to establish the effect of a $3 \mathrm{~d}$ high-F diet on the thermic, cardiovascular and metabolic responses to a simple glucose load. The results show that a high-F diet eaten for $3 \mathrm{~d}$ causes changes in the response to glucose ingestion. There is an increased thermic response and the plasma insulin response is also increased with a degree of insulin resistance being produced in these healthy subjects. The altered insulin and heart rate responses seen after the high-F diet are of a similar nature to the responses to food ingestion seen after a $48 \mathrm{~h}$ fast (Gallen \& Macdonald, 1990).

This study was supported by a Project Grant from the Wellcome Trust. The authors are grateful to Ms Heather Parkin for measuring the plasma catecholamines, and Mr Colin Selby (City Hospital, Nottingham) for measuring plasma insulin. 
Acheson, K. J., Ravussin, E., Wahren, J. \& Jequier, E. (1984). The thermic effect of glucose in man. Journal of Clinical Investigation 74, 1572-1580.

Alban-Davies, H. J., Baird, I., Fowler, J., Mills, I. H., Baille, J. E., Ratten, S. \& Howard, A. N. (1989). Metabolic responses to low- and very-low-calorie diets. American Journal of Clinical Nutrition 49, 745-751.

Andres, R., Zierler, K. I., Anderson, H. M., Stainsby, W. N., Cader, G., Ghrayyib, A. \& Lilienthal, J. L. (1954). Measurement of blood flow in human limbs; detailed notes on the theory of indicator-dilution and intravascular injection. Journal of Clinical Investigation 33, 482-504.

Elia, M. \& Livesey, G., (1988). Theory and validity of indirect calorimetry during net lipid synthesis. American Journal of Clinical Nutrition 47, 591-604.

Fagan, T., Sawyer, P. R., Gourley, L. A., Lee, J. T. \& Gaffney, T. E. (1986). Postprandial alteration in haemodynamics and blood pressure in normal subjects. American Journal of Cardiology 58, 636-641.

Fellows, I. W. \& Macdonald, I. A. (1985). An automated method for the measurement of oxygen consumption and carbon djoxide excretion in man. Clinical Physics and Physiological Measurement 6, 349-355.

Flatt, J. P. (1978). The biochemistry of energy expenditure. In Recent Advances in Obesity, vol. 2, pp. 211-218 [G. A. Bray, editor]. London: Newman.

Gallen, I. W. \& Macdonald, I. A. (1990). The effect of $48 \mathrm{~h}$ fast on the physiological responses to food ingestion in normal-weight women. British Journal of Nutrition 63, 53-64.

Lloyd, B., Burrin, J., Smythe, P. \& Alberti, K. G. M. M. (1978). Enzymic fluorimetric continuous flow assays for blood glucose, lactate, pyruvate, alanine, glycerol and 3-hydroxybutyrate. Clinical Chemistry 24, 1724-1729.

Lusk, G. (1924). Animal calorimetry. Analysis of the oxidation of mixtures of carbohydrate and fat. Journal of Biological Chemistry 59, 41-42.

Macdonald, I. A. \& Lake, D. M. (1985). An improved technique for extracting catecholamines from body fluids. Journal of Neuroscience Methods 13, 239-248.

McGuire, E. A. H., Helderman, J. H., Tobin, J. D., Andres, R. \& Berman, M. (1976). Effects of arterial versus venous sampling on analysis of glucose kinetics in man. Journal of Applied Physiology 41, 565-573.

Randle, P. J., Garland, P. B., Hales, C. N. \& Newsholme, E. A. (1963). The glucose fatty-acid cycle: its role in insulin sensitivity and the metabolic disturbances of diabetes mellitus. Lancet $\mathbf{i}, 785-789$.

Thiebaud, D., DeFronzo, R. A., Jacot, E., Golay, A., Acheson, K., Maeder, E., Jequier, E. \& Felber, J.-P. (1982). Effect of long-chain triglyceride infusion on glucose metabolism in man. Metabolism 31, 1128-1136.

Thulin, L. \& Samnegard, H. (1978). Circulatory effects of gastrointestinal hormones and related peptides. Acta Chirurgica Scandinavica 144, Suppl. 482, 73-74.

Webb, P. (1986). 24-hour energy expenditure and the menstrual cycle. American Journal of Clinical Nutrition 44, 614-619.

Weir, J. B. de V. (1949). New methods for calculating metabolic rate with special reference to protein metabolism. Journal of Physiology 109, 1-9.

Welle, S. L., Lilavivathana, U. \& Campbell, R. G. (1980). Increased plasma norepinephrine concentrations and metabolic rate following glucose ingestion in man. Metabolism 29, 806-809.

Whitney, R. J. (1953). Measurement of volume changes in human limbs. Journal of Physiology 121, 1-27. 